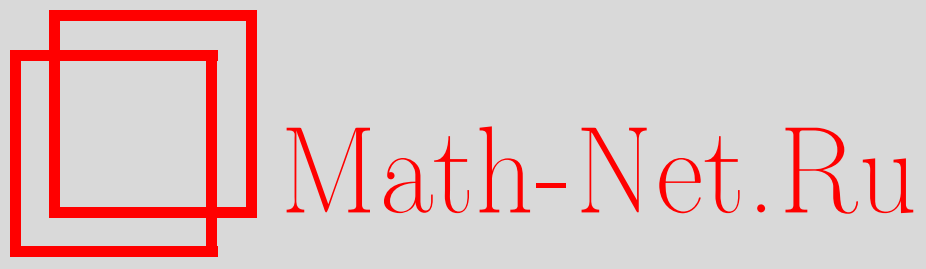

С. А. Кругляк, А. В. Ройтер, Локально скалярные представления графов в категории гильбертовых пространств, Функи. анализ и его прил., 2005, том 39, выпуск 2, 13-30

DOI: https://doi.org/10.4213/faa37

Использование Общероссийского математического портала MathNet.Ru подразумевает, что вы прочитали и согласны с пользовательским соглашением

http: //www . mathnet.ru/rus/agreement

Параметры загрузки:

IP: 54.174 .149 .18

26 апреля 2023 г., 14:23:35

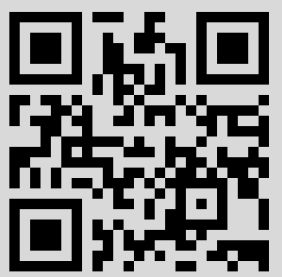


Функииональньй анализ и его приложения

2005, т. 39, вып. 2, с. 13-30

УДК 519.1

\title{
Локально скалярные представления графов в категории гильбертовых пространств
}

\author{
(C) 2005. С. А. Кругляк, А. В. РойтеР
}

\section{Введение}

В работе [1] авторы писали: «В последнее время стало ясно, что целый ряд задач линейной алгебры допускает единую формулировку, и в этой общей формулировке возникают общие эффективные методы исследования таких задач. Интересно, что эти методы оказываются связанными с такими понятиями, как группа Кокстера-Вейля и схемы Дынкина».

Одной из таких задач была задача о представлениях колчанов [2] (см. также [3-5]). По теореме Габриеля связный колчан имеет конечный представленческий тип тогда и только тогда, когда соответствующий ему неориентированный граф есть схема Дынкина. Объяснению этого факта (полученного П. Габриелем а posteriori) послужили функторы Кокстера, введенные в [1].

В дальнейшем теория представлений колчанов (в категории конечномерных векторных пространств) получила широкое развитие [6-10].

Можно рассматривать представления колчанов и в метрических, в частности гильбертовых пространствах [11-13]. Однако при напрашивающихся естественных определениях задача классификации представлений колчанов (за исключением колчанов, каждая связная компонента которых есть точка либо пара точек и стрелка между ними) в таких категориях оказывается «дикой» (в смысле [14], т. е. содержит в себе задачу об унитарной классификации произвольного линейного оператора или пары самосопряженных операторов).

Ниже мы вводим ограничение локальной скалярности на представления графа (или колчана) в категории гильбертовых пространств, после чего возникает теория, близкая к [1-10], хотя появляются и специфические для гильбертовых пространств аспекты.

Отметим, что в некотором частном случае (см. пример 3) задача классификации локально скалярных представлений в других терминах изучалась в $[16,17]$.

$\mathrm{B} \S \S 2,3$ для категории локально скалярных представлений графа в категории гильбертовых пространств вводятся функторы четных и нечетных отражений Кокстера. В 44 с их помощью доказывается аналог теоремы Габриеля.

\section{§1. Основные определения}

Пусть $\mathscr{H}$ - категория гильбертовых пространств, объектами которой являются сепарабельные гильбертовы пространства (конечномерные либо бесконечномерные), а морфизмами - ограниченные операторы. Каждому $\varphi \in \mathscr{H}(A, B)$ однозначно сопоставлен $\varphi^{*} \in \mathscr{H}(B, A)$. 
Следуя Габриелю [2], будем называть ориентированные графы колчанами, оставив термин «граф» для неориентированных графов. В обоих случаях будем допускать петли, кратные стрелки и ребра.

Таким образом, колчан $Q$ состоит из двух множеств $Q_{v}$ (вершин) и $Q_{a}$ (стрелок) и двух отображений $t$ и $h$ из $Q_{a}$ в $Q_{v}$, ставящих в соответствие каждой стрелке $\alpha \in Q_{a}$ ее начало (tail) $t(\alpha)$ и конец (head) $h(\alpha)$.

Граф $G$ состоит из множеств $G_{v}$ (вершин) и $G_{e}$ (ребер) и отображения $\varepsilon$ из $G_{e}$ в множество одноэлементных и двухэлементных подмножеств множества $G_{v} .(\varepsilon(\gamma)$ состоит из вершин, соединенных ребром $\gamma$.$) Каждому колчану$ $Q$ естественным образом сопоставляется его граф $G=G(Q)$.

Представление $\pi$ колчана $Q$ в произвольной категории $K$ сопоставляет каждой вершине $a \in Q_{v}$ объект $\pi(a) \in K$ и каждой стрелке $\alpha \in Q_{a}$ морфизм $\pi(\alpha) \in K(A, B)$, где $A=\pi(t(\alpha)), B=\pi(h(\alpha))$.

В [1-10] $K=\bmod k$, где $k$ - поле. Представления колчана $Q$ над полем $k$ образуют категорию $\operatorname{Rep}(Q, k)$.

Если $\pi$ - представление колчана $Q$ в $\mathscr{H}$, то всякой стрелке $\alpha: a \rightarrow b$ соответствует, кроме оператора $\pi(\alpha): A \rightarrow B$, оператор $\pi^{*}(\alpha): B \rightarrow A$. Поэтому в категории $\mathscr{H}$ естественно определить представление $\pi$ графа $G=G(Q)$, которое сопоставляет каждой вершине $a \in G_{v}$ объект $\pi(a)=H_{a} \in \mathscr{H}$ и каждому ребру $\gamma \in G_{e}$, у которого $\varepsilon(\gamma)=\{a, b\}$, - пару взаимно сопряженных линейных операторов $\pi(\gamma)=\left\{\Gamma_{a b}, \Gamma_{b a}\right\}$, где $\Gamma_{a b}: H_{b} \rightarrow H_{a}$, а при $\varepsilon(\gamma)=\{a\}-$ пару взаимно сопряженных операторов $\pi(\gamma)=\left\{\Gamma_{a a}, \Gamma_{a a}^{*}\right\}$.

Следовательно, можно отождествить представления колчана $Q$ и графа $G=$ $G(Q)$ в категории $\mathscr{H}$. Мы предпочтем в этой работе рассматривать в гильбертовых пространствах представления графов, но все результаты естественно перефразируются для колчанов.

Построим категорию $\operatorname{Rep}(G, \mathscr{H})$ представлений графа $G$ в $\mathscr{H}$.

Морфизмом $C: \pi \rightarrow \tilde{\pi}$ будем считать семейство $\left\{C_{a}\right\}_{a \in G_{v}}$ операторов $C_{a}: \pi(a)$ $\rightarrow \tilde{\pi}(a)$, таких, что диаграммы

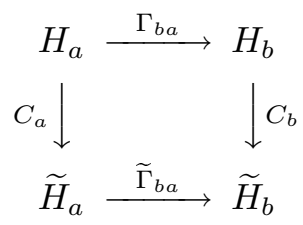

коммутативны, т. е.

$$
C_{b} \Gamma_{b a}=\widetilde{\Gamma}_{b a} C_{a} .
$$

Можно показать, что операторы $C_{a}$, осуществляющие эквивалентность двух представлений, можно выбрать унитарными (см., например, [15]), т. е. эквивалентные объекты категории $\operatorname{Rep}(G, \mathscr{H})$ унитарно эквивалентны.

Представление $\pi$ графа $G$ конечномерно, если $\operatorname{dim} \pi(a)<\infty$ для всех $a \in G_{v}$.

Граф $G_{0}$, состоящий из одной петли, имеет бесконечномерные неразложимые (в категории $\left.\operatorname{Rep}\left(G_{0}, \mathscr{H}\right)\right)$ представления, например представление, заданное оператором одностороннего сдвига и сопряженным к нему.

Будем называть представление $\pi$ графа $G$ дискретным, если оно распадается в прямую сумму (конечную либо бесконечную) конечномерных представлений. Носитель $G_{v}^{\pi}=G^{\pi}$ представления $\pi$ есть множество $\left\{a \in G_{v} \mid \pi(a) \neq 0\right\}$. 
Обозначим через $M_{a}$ множество вершин, соединенных с вершиной $a$ ребром; положим $\bar{M}_{a}=\left\{\gamma \in G_{e} \mid a \in \varepsilon(\gamma)\right\}$, и пусть $M(X)=\left(\bigcup_{a \in X} M_{a}\right) \backslash X$, если $X \subset G_{v}$.

Представление $\pi$ точное, если $G^{\pi}=G_{v}$.

Пусть $\pi \in \operatorname{Rep}(G, \mathscr{H})$. Если $\varepsilon(\gamma)=\{g, b\}$, положим $A(\gamma, g)=\Gamma_{b g}^{*} \Gamma_{b g}$, а при $\varepsilon(\gamma)=\{g\}$ положим $A(\gamma, g)=\Gamma_{g g}^{*} \Gamma_{g g}+\Gamma_{g g} \Gamma_{g g}^{*}$ и

$$
A_{g}=\sum_{\gamma \in \bar{M}_{g}} A(\gamma, g) \text {. }
$$

Тогда $A_{g}$ - самосопряженный оператор в пространстве $H_{g}=\pi(g)$. Если $\bar{M}_{g}=\varnothing$, будем считать, что $A_{g}=0$. Заметим, что если $G$ не содержит петель и кратных ребер, то

$$
A_{g}=\sum_{b \in M_{g}} \Gamma_{g b} \Gamma_{b g}
$$

Представление $\pi$ будем называть локально скалярным, если все операторы $A_{g}$ скалярны, $A_{g}=\alpha_{g} I_{H_{g}}$, где $I_{H_{g}}$ - тождественный оператор в пространстве $H_{g}$; поскольку $A_{g}$ - положительные операторы, то $\alpha_{g} \geqslant 0$.

Если $\pi(b)=0$ для всех $b \in M_{a}, b \neq a$, а $\pi(a) \neq 0$, то, очевидно, $\alpha_{a}=0$. Полную подкатегорию в $\operatorname{Rep}(G, \mathscr{H})$, состоящую из локально скалярных объектов, обозначим через $\operatorname{Rep}(G)$.

Обозначим через $V_{G}$ линейное вещественное пространство, состоящее из наборов $x=\left(x_{a}\right)$ вещественных чисел $x_{a}\left(a \in G_{v}\right)$; элементы $x$ из $V_{G}$ будем называть $G$-векторами (или просто векторами). Будем отождествлять $G$-вектор $x=\left(x_{a}\right)$ с (вещественной) функцией $x=x(a)$, принимающей в каждой вершине $a \in G_{v}$ значение $x_{a}$. Введем следующие обозначения: $V_{G}^{+}=\left\{x \in V_{G} \mid x>0\right\}$, $Z_{G}=\left\{x \in V_{G} \mid x(g) \in \mathbb{N}_{0}\right.$ для всех $\left.g \in G_{v}\right\}\left(\mathbb{N}_{0}=\mathbb{N} \cup\{0\}\right), Z_{G}^{+}=Z_{G} \cap V_{G}^{+}$.

$G$-вектор $d(a)=\operatorname{dim} \pi(a)$ есть размерность конечномерного представления $\pi \in \operatorname{Rep}(G, \mathscr{H}) ;$ если $A_{g}=f(g) I_{H_{g}}$ для $g \in G_{v}$, то $f(g)$ будем называть характером локально скалярного представления $\pi$, а само $\pi$ назовем $f$-nредставлением. Характер определен однозначно на носителе $G^{\pi}$ представления $\pi$ (и неоднозначно вне носителя). Если $G_{v}=G^{\pi}$, то характер представления определен однозначно и обозначается через $f_{\pi}$. В общем случае обозначим через $\left\{f_{\pi}\right\}$ множество характеров представления $\pi$.

Для графов могут существовать как бесконечномерные, так и конечномерные неразложимые представления, и размерности последних могут быть не ограничены. При этом даже в простейших случаях имеется бесконечно много неразложимых представлений в фиксированной размерности, отвечающих различным характерам.

Будем говорить, что $G$ (локально скалярно) конечно представим в $\mathscr{H}$, если все его локально скалярные представления дискретны, размерности его неразложимых локально скалярных представлений ограничены в совокупности и в каждой размерности число неразложимых представлений с данным характером конечно.

Мы в дальнейшем докажем, что связный конечный граф конечно представим, если и только если он есть схема Дынкина $\left(A_{n}, D_{n}, E_{6}, E_{7}, E_{8}\right)$, и в этом случае его неразложимые представления однозначно определяются размерностью и значениями характера на носителе представления. 
ПримеР 1. Произвольный граф $G$ имеет локально скалярное представление $\pi$ в размерности $d(a) \equiv 1$. Положим $\pi(a)=\mathbb{C} e_{a},\left(e_{a}, e_{a}\right)=1$ и $\pi\left(\gamma_{a b}\right)=$ $\left\{\Gamma_{b a}, \Gamma_{a b}\right\}$, где $\Gamma_{b a} e_{a}=e_{b}$ и $\Gamma_{a b} e_{b}=e_{a}\left(\varepsilon\left(\gamma_{a b}\right)=\{a, b\}\right)$. Представление $\pi$ неразложимо тогда и только тогда, когда $G$ связен.

ПримеР 2. $G_{0}$ состоит из одной вершины $a$ и одной петли $\gamma$. Пусть $\pi-$ локально скалярное представление графа $G_{0}$, для которого $\pi(a)=H, \pi(\gamma)=$ $\left\{\Gamma, \Gamma^{*}\right\}, \Gamma: H \rightarrow H, f(a)=\alpha$, т. е. $\Gamma \Gamma^{*}+\Gamma^{*} \Gamma=\alpha I_{H}$. В [14] показано, что все неразложимые локально скалярные представления графа $G_{0}$ не более чем двумерны; при фиксированном положительном $\alpha$ в размерности 2 неразложимые представления зависят от двух непрерывных параметров, так что граф $G_{0}$ не является конечно представимым.

Приведем пример недискретного представления графа $G_{0}$, которое разложимо, хотя не может быть разложено в прямую сумму неразложимых представлений (а только в их интеграл). Рассмотрим гильбертово пространство $H$ с (ортонормированным) базисом $\left\{e_{i}\right\}, i \in \mathbb{Z}$, и в нем оператор $\Gamma\left(e_{i}\right)=e_{i+1}$ $\left(\Gamma^{*} \Gamma+\Gamma \Gamma^{*}=2 I_{H}\right)$. Тогда $H$ не содержит конечномерных инвариантных подпространств.

ПримеР 3. Задача (унитарной) классификации локально скалярных представлений графа $G_{n}$

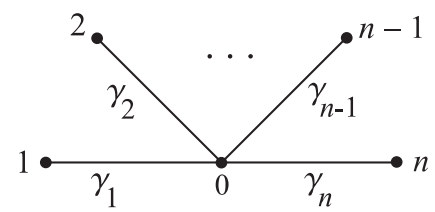

фактически рассматривалась (в других терминах) в работах $[16-19]$. В $[16,17]$ изучалась задача классификации с точностью до унитарной эквивалентности наборов ортопроекторов $P_{1}, \ldots, P_{n}$ (в сепарабельном гильбертовом пространстве $H$ ), таких, что $\sum_{k=1}^{n} P_{k}=\alpha I_{H}$, и, в частности, задача описания множества $\Sigma_{n}$ тех вещественных чисел $\alpha$, для которых найдется хотя бы один набор таких ортопроекторов (в ненулевом пространстве).

Сопоставляя ортопроектору $P_{i}$ пространство $H_{i}=\operatorname{Im} P_{i}$ и естественное вложение (изометрию) $\Gamma_{i}: H_{i} \rightarrow H$, мы получим локально скалярное представление $\pi$ графа $G_{n}$, полагая $\pi(i)=H_{i}, i=1, \ldots, n, \pi(0)=H, \pi\left(\gamma_{i}\right)=\left\{\Gamma_{i}, \Gamma_{i}^{*}\right\}$. При этом $f(i)=1$ при $i \neq 0$ и $f(0)=\alpha\left(\Gamma_{i}^{*} \Gamma_{i}=I_{H_{i}}, P_{i}=\Gamma_{i} \Gamma_{i}^{*}\right.$ и $\left.\sum_{k=1}^{n} \Gamma_{k} \Gamma_{k}^{*}=\alpha I_{H}\right)$.

В $[18,19]$ для представлений графа $G_{n}$ построены функторы, строение и роль которых при описании представлений такая же, как функторов Кокстера в [1]. С помощью этих функторов в [17] получены полное описание множества $\Sigma_{n}$ и новые результаты о наборах ортопроекторов.

Как отмечалось выше, граф $G_{0}$ (петля) имеет бесконечномерные неразложимые представления в $\mathscr{H}$, но они не локально скалярны. Как следует из [17], граф $G_{n}$ имеет бесконечномерные неразложимые локально скалярные представления, если и только если $n>4$.

\section{§2. Функторы отражений}

Из примера 2 следует, что если граф $G$ содержит цикл, то этот граф не конечно представим в $\mathscr{H}$. Мы будем изучать вопрос о конечной представимости 
и поэтому ограничимся графами, не содержащими циклов: везде в дальнейшем $G$ - конечный связный граф без циклов (дерево).

Зафиксируем разложение множества $G_{v}$ вида $\stackrel{\circ}{G} \amalg \stackrel{\bullet}{G}$ (однозначное с точностью до перестановки $\stackrel{\circ}{G}$ и $\stackrel{\bullet}{G})$, такое, что для каждого $\alpha \in G_{e}$ одна из вершин из $\varepsilon(\alpha)$ лежит в $\stackrel{\circ}{G}$, а другая - в $\stackrel{\bullet}{G}$. Вершины множества $\stackrel{\circ}{G}$ назовем четными, а множества $\stackrel{\bullet}{G}$ - нечетными.

Для каждой вершины $a \in G_{v}$ обозначим через $\sigma_{a}$ линейное преобразование в пространстве $V_{G}$, задаваемое формулами $\left(\sigma_{a} x\right)_{b}=x_{b}$ при $b \neq a,\left(\sigma_{a} x\right)_{a}=$ $-x_{a}+\sum_{b \in M_{a}} x_{b}$. Будем называть $\sigma_{a}$ отражением в вершине $a$. Обозначим через $W$ группу преобразований пространства $V_{G}$, порожденную отражениями $\sigma_{a}$.

Зафиксируем нумерацию вершин графа $G$, занумеровав сначала нечетные, а затем четные вершины. Произведение отражений во всех нечетных вершинах обозначим через $\stackrel{\bullet}{c}$, а в четных - через $\stackrel{\circ}{c}$ (заметим, что отражения в нечетных (четных) вершинах между собой коммутируют). Преобразование Кокстера на $V_{G}$ есть $c=\stackrel{\bullet}{c} \cdot$, причем $c^{-1}=\stackrel{\bullet}{c} c ; \dot{c}(\stackrel{\circ}{c})$ будем называть нечетным (четным) преобразованием Кокстера.

Обозначим через $\stackrel{\bullet}{c}_{k}$ и $\stackrel{\circ}{c}_{k}$ композиции $k$ чередующихся преобразований $\stackrel{\bullet}{c}$ и $\stackrel{\circ}{c}$ : $\stackrel{\bullet}{c}_{k}=\underbrace{\ldots \stackrel{\circ}{*} c}_{k \text { раз }}, \stackrel{\circ}{c}_{k}=\underbrace{\cdots \bullet c}_{k \text { раз }}, k \in \mathbb{N}$ (в частности, $\stackrel{\bullet}{c}_{2}=c, \stackrel{\circ}{c_{2}}=c^{-1})$.

Пусть $X \subset G_{v}$. Обозначим через $\operatorname{Rep}_{\circ}(G, X)$ полную подкатегорию локально скалярных представлений $\pi$ в категории $\operatorname{Rep}(G)$, для которых $G^{\pi}=X$, а характер положителен на $G^{\pi} \cap \stackrel{\circ}{G}$. Пусть $(M(X))^{\circ}=M(X) \cap \stackrel{\circ}{G}$ и $\delta:(M(X))^{\circ} \rightarrow \mathbb{R}^{+}$. Не исключается и случай, когда $(M(X))^{\circ}=\varnothing$.

Построим функтор

$$
\stackrel{\circ}{F}_{X, \delta}: \operatorname{Rep}_{\circ}(G, X) \rightarrow \operatorname{Rep}(G) .
$$

Из дальнейшего следует, что локальная скалярность представлений существенна для построения функтора $\stackrel{\circ}{F}_{X, \delta}\left(\right.$ если $(M(X))^{\circ}=\varnothing$, будем обозначать этот функтор через $\stackrel{\circ}{F}_{X}$, а если $X=G_{v}$, то через $\left.\stackrel{\circ}{F}_{G}\right)$.

Если гильбертовы пространства $H$ и $\widehat{H}$ разложены в ортогональную сумму подпространств, $H=H^{(1)} \oplus \cdots \oplus H^{(n)}, \widehat{H}=\widehat{H}^{(1)} \oplus \cdots \oplus \widehat{H}^{(m)}$, то произвольный линейный оператор $A: H \rightarrow \widehat{H}$ может быть записан в виде матрицы $A=\left[A_{i j}\right]_{j=1, \ldots, n}^{i=1, \ldots, m}$, где операторы $A_{i j}$ действуют из пространства $H^{(j)}$ в пространство $\widehat{H}^{(i)}$. Такие матрицы перемножаются по обычным правилам умножения блочных матриц.

Пусть $\pi \in \mathrm{Ob} \operatorname{Rep}_{\circ}(G, X)$ и $a \in X^{\circ} \cup(M(X))^{\circ}$. Зафиксируем $f \in\left\{f_{\pi}\right\}$, считая, что $f(b)=\delta(b)$ при $b \in(M(X))^{\circ}$.

Из условий локальной скалярности в вершине $а$ следует, что оператор

$$
\Gamma_{a}^{*}=\left[\frac{1}{\sqrt{\alpha_{a}}} \Gamma_{a g_{1}}, \ldots, \frac{1}{\sqrt{\alpha_{a}}} \Gamma_{a g_{k}}\right], \quad \alpha_{a}=f(a),
$$

действующий из пространства $H^{(a)}=H_{g_{1}} \oplus \cdots \oplus H_{g_{k}}$ (сумма ортогональная, $\left.\left\{g_{1}, \ldots, g_{k}\right\}=M_{a}\right)$ в пространство $H_{a}$, обладает свойством $\Gamma_{a}^{*} \Gamma_{a}=I_{H_{a}}$ (т. е. 
оператор $\Gamma_{a}$ есть изометрия пространства $H_{a}$ в пространство $\left.H^{(a)}\right)$. Пусть $\widehat{H}_{a}$ ортогональное дополнение к $\operatorname{Im} \Gamma_{a}$. Тогда если $\Delta_{a}-$ естественное вложение пространства $\widehat{H}_{a}$ в $H^{(a)}$ и

$$
\Delta_{a}^{*}=\left[\frac{1}{\sqrt{\alpha_{a}}} \Delta_{a g_{1}}, \ldots, \frac{1}{\sqrt{\alpha_{a}}} \Delta_{a g_{k}}\right],
$$

то оператор

$$
U_{a}^{*}=\left[\begin{array}{ccc}
\frac{1}{\sqrt{\alpha_{a}}} \Gamma_{a g_{1}} & \ldots & \frac{1}{\sqrt{\alpha_{a}}} \Gamma_{a g_{k}} \\
\frac{1}{\sqrt{\alpha_{a}}} \Delta_{a g_{1}} & \ldots & \frac{1}{\sqrt{\alpha_{a}}} \Delta_{a g_{k}}
\end{array}\right]
$$

есть унитарный оператор, $U_{a}^{*}: H^{(a)} \rightarrow H_{a} \oplus \widehat{H}_{a}, U_{a}^{*} U_{a}=I_{H_{a} \oplus \widehat{H}_{a}}, U_{a} U_{a}^{*}=I_{H^{(a)}}$.

Рассмотрим операторы $\Delta_{g_{t}, a}: \widehat{H}_{a} \rightarrow H_{g_{t}}$ и $\Delta_{a, g_{t}}=\Delta_{g_{t}, a}^{*}: H_{g_{t}} \rightarrow \widehat{H}_{a}$.

Положим $\stackrel{\circ}{\pi}(a)=\widehat{H}_{a}$ для $a \in X^{\circ} \cup(M(X))^{\circ}$ и $\stackrel{\circ}{\pi}(b)=H_{b}$ для остальных вершин, и пусть $\stackrel{\circ}{\pi}\left(\gamma_{a b}\right)=\left\{\Delta_{b a}, \Delta_{a b}\right\}$ для $a \in X^{\circ} \cup(M(X))^{\circ}, b \in M_{a}$ и $\stackrel{\circ}{\pi}\left(\gamma_{k l}\right)=\pi\left(\gamma_{k l}\right)$ для остальных ребер. В результате мы получим представление $\stackrel{\circ}{\pi} \in \operatorname{Ob} \operatorname{Rep}(G, \mathscr{H})$.

Покажем, что представление $\stackrel{\circ}{\pi}$ будет локально скалярным.

Так как $U_{a}$ - унитарные операторы, то

$$
\sum_{b \in M_{a}} \Delta_{a b} \Delta_{b a}=\alpha_{a} I_{\widehat{H}_{a}} \quad \text { и } \quad \Gamma_{b a} \Gamma_{a b}+\Delta_{b a} \Delta_{a b}=\alpha_{a} I_{H_{b}}
$$

при $a \in X^{\circ} \cup(M(X))^{\circ}, b \in M_{a}$.

Из соотношений (3) для вершин $b \in X^{\bullet}=X \cap \dot{G}$ следует, что

$$
\sum_{a \in M_{b}} \Delta_{b a} \Delta_{a b}=\left(-\alpha_{b}+\sum_{a \in M_{b}} \alpha_{a}\right) I_{H_{b}} .
$$

Поэтому если мы положим $\stackrel{\circ}{f}(a)=f(a)$ для вершин $a \in X^{\circ}$, а для $b \in X^{\bullet}$ определим $\stackrel{\circ}{f}(b)$ как сумму значений функции $f$ в соседних с $b$ вершинах минус ее значение в вершине $b$, то $\stackrel{\circ}{f} \in\left\{f_{\circ}\right\}$.

Положим $\stackrel{\circ}{F}_{X, \delta}(\pi)=\stackrel{\circ}{\pi}$. Отметим, что при различных $\delta$ мы будем получать различные локально скалярные представления.

Определим действие $\stackrel{\circ}{F}_{X, \delta}$ на морфизмах категории $\operatorname{Rep}_{\circ}(G, X)$. Пусть $\pi, \widetilde{\pi} \in$ $\mathrm{ObRep}_{\circ}(G, X)$ и $C=\left\{C_{a}\right\}_{a \in G_{v}}: \pi \rightarrow \tilde{\pi}-$ морфизм в $\operatorname{Rep}_{\circ}(G, X)$. При $b \notin$ $X^{\circ} \cup(M(X))^{\circ}$ положим $\stackrel{\circ}{C_{b}}=C_{b}$.

Пусть $a \in(M(X))^{\circ} \cup X^{\circ}$ и $\alpha_{a}=f(a)>0, \widetilde{\alpha}_{a}=\widetilde{f}(a)>0$. Из равенств (1) для $b \in M_{a}$ следует, что $\widetilde{\Gamma}_{a b} C_{b} \Gamma_{b a}=C_{a} \Gamma_{a b} \Gamma_{b a}$; поэтому $\sum_{b \in M_{a}} \widetilde{\Gamma}_{a b} C_{b} \Gamma_{b a}=$ $\sum_{b \in M_{a}} C_{a} \Gamma_{a b} \Gamma_{b a}=\alpha_{a} C_{a}$ и, следовательно,

$$
C_{a}=\frac{1}{\alpha_{a}} \sum_{b \in M_{a}} \widetilde{\Gamma}_{a b} C_{b} \Gamma_{b a}
$$


$\mathrm{C}$ другой стороны, из (1) для $b \in M_{a}$ следует, что $\widetilde{\Gamma}_{a b} C_{b} \Gamma_{b a}=\widetilde{\Gamma}_{a b} \widetilde{\Gamma}_{b a} C_{a}, \mathrm{a}$ значит, $\sum_{b \in M_{a}} \widetilde{\Gamma}_{a b} C_{b} \Gamma_{b a}=\left(\sum_{b \in M_{a}} \widetilde{\Gamma}_{a b} \widetilde{\Gamma}_{b a}\right) C_{a}=\widetilde{\alpha}_{a} C_{a}$ и

$$
C_{a}=\frac{1}{\widetilde{\alpha}_{a}} \sum_{b \in M_{a}} \widetilde{\Gamma}_{a b} C_{b} \Gamma_{b a} .
$$

Поэтому $C_{a}=0$ при $\alpha_{a} \neq \widetilde{\alpha}_{a}$. Положим $\stackrel{\circ}{C}_{a}=0$ при $\alpha_{a} \neq \widetilde{\alpha}_{a}$ и

$$
\stackrel{\circ}{C}_{a}=\frac{1}{\alpha_{a}} \sum_{b \in M_{a}} \widetilde{\Delta}_{a b} C_{b} \Delta_{b a}
$$

при $\alpha_{a}=\widetilde{\alpha}_{a}$. Покажем, что коммутативна диаграмма

$$
\begin{aligned}
& \widehat{H}_{a} \stackrel{\Delta_{b a}}{\longrightarrow} \widehat{H}_{b}=H_{b} \\
& \stackrel{\circ}{C_{a}} \downarrow \quad \downarrow \stackrel{\circ}{C_{b}}=C_{b} \\
& \widehat{\widetilde{H}}_{a} \stackrel{\tilde{\Delta}_{b a}}{\longrightarrow} \widehat{\widetilde{H}}_{b}=\widetilde{H}_{b}
\end{aligned}
$$

где $b \in M_{a}, a \in \stackrel{\circ}{G}$, т. е. $C_{b} \Delta_{b a}=\widetilde{\Delta}_{b a} \stackrel{\circ}{C}_{a}$. В силу равенства $(7)$

$$
\widetilde{\Delta}_{b a} \stackrel{\circ}{C}_{a}=\widetilde{\Delta}_{b a} \cdot \frac{1}{\alpha_{a}} \sum_{g \in M_{a}} \widetilde{\Delta}_{a g} C_{g} \Delta_{g a} .
$$

Из унитарности операторов $U_{a}^{*}, \widetilde{U}_{a}^{*}$, записанных в форме $(2)$, следует, что

$$
\widetilde{\Gamma}_{b a} \widetilde{\Gamma}_{a g}+\widetilde{\Delta}_{b a} \widetilde{\Delta}_{a g}=0 \text { при } b \neq g \quad \text { и } \quad \widetilde{\Gamma}_{b a} \widetilde{\Gamma}_{a b}+\widetilde{\Delta}_{b a} \widetilde{\Delta}_{a b}=\alpha_{a} I_{\widetilde{H}_{b}} ;
$$

поэтому

$$
\begin{aligned}
\widetilde{\Delta}_{b a} \stackrel{\circ}{C}_{a} & =\frac{1}{\alpha_{a}} \sum_{g \in M_{a}, g \neq b}\left[-\widetilde{\Gamma}_{b a} \widetilde{\Gamma}_{a g} C_{g} \Delta_{g a}\right]+\left[I_{\widetilde{H}_{b}}-\frac{1}{\alpha_{a}} \widetilde{\Gamma}_{b a} \widetilde{\Gamma}_{a b}\right] \cdot C_{b} \Delta_{b a} \\
& =C_{b} \Delta_{b a}-\frac{1}{\alpha_{a}} \sum_{g \in M_{a}, g \neq b} \widetilde{\Gamma}_{b a} \widetilde{\Gamma}_{a g} C_{g} \Delta_{g a}-\frac{1}{\alpha_{a}} \widetilde{\Gamma}_{b a} \widetilde{\Gamma}_{a b} C_{b} \Delta_{b a} .
\end{aligned}
$$

Вследствие соотношений (1) получаем, что

$$
\begin{aligned}
\widetilde{\Delta}_{b a} \stackrel{\circ}{C}_{a} & =C_{b} \Delta_{b a}-\frac{1}{\alpha_{a}} \sum_{g \in M_{a}, g \neq b} C_{b} \Gamma_{b a} \Gamma_{a g} \Delta_{g a}-\frac{1}{\alpha_{a}} C_{b} \Gamma_{b a} \Gamma_{a b} \Delta_{b a} \\
& =C_{b} \Delta_{b a}-\frac{1}{\alpha_{a}} C_{b} \Gamma_{b a} \cdot \sum_{g \in M_{a}} \Gamma_{a g} \Delta_{g a} .
\end{aligned}
$$

Последняя сумма равна 0 из-за ортогональности блок-строк унитарного оператора (2). Поэтому $\widetilde{\Delta}_{b a} \stackrel{\circ}{C}_{a}=C_{b} \Delta_{b a}$ и диаграмма (8) коммутативна.

Коммутативность двойственной диаграммы проверяется аналогично; следовательно, $\stackrel{\circ}{C}=\left\{\stackrel{\circ}{C}_{g}\right\}_{g \in G_{v}}$ действительно есть морфизм категории $\operatorname{Rep}(G, \mathscr{H})$.

Покажем, что $\stackrel{\circ}{F}_{X, \delta}$ сохраняет произведения морфизмов и тождественные морфизмы. Пусть $C=\left\{C_{g}\right\}: \pi \rightarrow \widetilde{\pi}$ и $D=\left\{D_{g}\right\}: \widetilde{\pi} \rightarrow \widetilde{\widetilde{\pi}}-$ морфизмы в 
категории $\operatorname{Rep}_{\circ}(G, X)$. Из коммутативности диаграмм

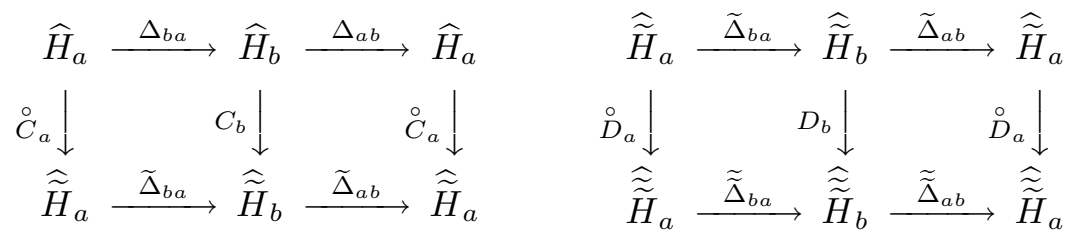

следует коммутативность диаграммы

$$
\begin{aligned}
& \widehat{H}_{a} \stackrel{\Delta_{b a}}{\longrightarrow} \widehat{H}_{b} \stackrel{\Delta_{a b}}{\longrightarrow} \widehat{H}_{a} \\
& \stackrel{\circ}{D}_{a} \stackrel{\circ}{C}_{a} \downarrow \quad D_{b} C_{b} \downarrow \quad \stackrel{\circ}{D}_{a} \stackrel{\circ}{C}_{a} \downarrow
\end{aligned}
$$

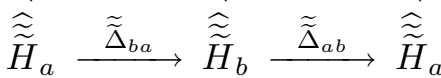

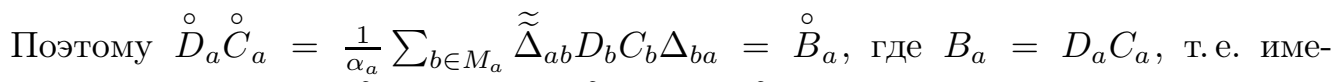
ет место равенство $\stackrel{\circ}{F}_{X, \delta}(D C)=\stackrel{\circ}{F}_{X, \delta}(D) \cdot \stackrel{\circ}{F}_{X, \delta}(C)$. Если $C=\left\{I_{H_{k}}\right\}_{k \in G_{v}}$, то $\stackrel{\circ}{C}_{b}=C_{b}=I_{H_{b}}$ при $b \notin(M(X))^{\circ} \cup X^{\circ}$, а при $a \in(M(X))^{\circ} \cup X^{\circ}$ имеем $\stackrel{\circ}{C}_{a}=\frac{1}{\alpha_{a}} \sum_{b \in M_{a}} \Delta_{a b} I_{H_{b}} \Delta_{b a}=I_{H_{a}}$, т. е. $\stackrel{\circ}{F}_{X, \delta}\left(\left\{I_{H_{k}}\right\}\right)=\left\{I_{H_{k}}\right\}$.

Итак, мы построили функтор $\stackrel{\circ}{F}_{X, \delta}$. Аналогично строится функтор

$$
\dot{F}_{X, \delta}: \operatorname{Rep}_{\bullet}(G, X) \rightarrow \operatorname{Rep}(G) .
$$

Пусть теперь $\pi$ - конечномерное представление графа $G$. Легко подсчитать, что при действии функтора $\stackrel{\circ}{F}_{X, \delta}$ функция размерности $d(a)$ меняется следующим образом: представление $\stackrel{\circ}{F}_{X, \delta}(\pi)$ имеет размерность $\stackrel{\circ}{c}(d)$,

$$
\stackrel{\circ}{c}(d)(a)= \begin{cases}-d(a)+\sum_{b \in M_{a}} d(b) & \text { при } a \in \stackrel{\circ}{G}, \\ d(a) & \text { при } a \in \stackrel{\bullet}{G},\end{cases}
$$

а характер представления $\stackrel{\circ}{F}_{G}(\pi)$ есть $\stackrel{\bullet}{c}(f)$,

$$
\dot{c}(f)(a)= \begin{cases}-f(a)+\sum_{b \in M_{a}} f(b) & \text { при } a \in \dot{G}, \\ f(a) & \text { при } a \in \stackrel{\circ}{G .}\end{cases}
$$

Аналогично находится размерность $\stackrel{\bullet}{c}(d)$ представления $\dot{F}_{X, \delta}(\pi)$ и характер представления $\dot{\bullet}_{G}(\pi)$.

При $d \in Z_{G}^{+}, f \in V_{G}^{+}$рассмотрим полную подкатегорию $\operatorname{Rep}(G, d, f)$ в $\operatorname{Rep}(G)$, $\mathrm{Ob} \operatorname{Rep}(G, d, f)=\left\{\pi \mid d=d(\pi), f \in\left\{f_{\pi}\right\}\right\}$. Все представления $\pi$ из $\operatorname{Rep}(G, d, f)$ имеют общий носитель $X_{d}=G^{\pi}=\left\{a \in G_{v} \mid d(a) \neq 0\right\}$.

Будем рассматривать эти категории при условии, что $(d, f) \in S=\{(d, f) \in$ $\left.Z_{G}^{+} \times V_{G}^{+} \mid d(a)+f(a)>0, a \in G_{v}\right\}$. 
Напомним, что размерность представления определяется однозначно, а характер, вообще говоря, нет; поэтому объекты $\operatorname{Rep}(G, d, f)$ можно рассматривать как пары $(\pi, f), f \in\left\{f_{\pi}\right\}$.

Пусть $\widetilde{\operatorname{Rep}}(G, d, f) \subset \operatorname{Rep}(G, d, f)$ - полная подкатегория, объекты которой есть пары $(\pi, f)$ с представлениями $\pi$, неразложимыми в $\operatorname{Rep}(G, \mathscr{H})$. Пусть $\operatorname{Rep}_{\circ}(G, d, f) \subset \operatorname{Rep}(G, d, f)(\operatorname{Rep} .(G, d, f) \subset \operatorname{Rep}(G, d, f))-$ полная подкатегория, объекты которой есть пары $(\pi, f)$ с $f(a)>0$ при $a \in X^{\circ}=X \cap \stackrel{\circ}{G}$ (с $f(a)>0$ при $\left.a \in X^{\bullet}=X \cap \dot{G}\right)$. Естественным образом вводятся $\widetilde{\operatorname{Rep}}$ 。 $(G, d, f)$ и $\widetilde{\operatorname{Rep}} .(G, d, f)$.

Пусть $S_{\circ}=\left\{(d, f) \in S \mid f(a)>0\right.$ при $\left.a \in \stackrel{\circ}{X}_{d}\right\}, S_{\bullet}=\{(d, f) \in S \mid f(a)>0$ при $\left.a \in \dot{X}_{d}\right\}$. При $(d, f) \in S_{\circ}\left((d, f) \in S_{\bullet}\right)$ построим функторы четных и нечетных отражений

$$
\begin{aligned}
& \stackrel{\circ}{F_{d f}}: \operatorname{Rep}_{\circ}(G, d, f) \rightarrow \operatorname{Rep}_{\circ}\left(G, \stackrel{\circ}{c}(d), \stackrel{\circ}{f}_{d}\right), \quad \stackrel{\circ}{f}_{d}(a)= \begin{cases}\stackrel{\bullet}{c}(f)(a) & \text { при } a \in \dot{X}_{d}, \\
f(a) & \text { при } a \notin \dot{X}_{d},\end{cases} \\
& \dot{F}_{d f}: \operatorname{Rep}(G, d, f) \rightarrow \operatorname{Rep}\left(G, \dot{c}(d), \dot{f}_{d}\right), \quad \dot{f}_{d}(a)= \begin{cases}\stackrel{\circ}{c}(f)(a) & \text { при } a \in \stackrel{\circ}{X}_{d}, \\
f(a) & \text { при } a \notin \stackrel{\circ}{X}_{d} .\end{cases}
\end{aligned}
$$

Положим $\stackrel{\circ}{F}_{d f}(\pi, f)=\left(\stackrel{\circ}{\pi}, \stackrel{\circ}{f}_{d}\right)$, где $\stackrel{\circ}{\pi}=\stackrel{\circ}{F}_{X_{d}, \delta}(\pi)$ и $\stackrel{\circ}{F}_{d f}(\pi, f)=\stackrel{\bullet}{F}_{X_{d}, \delta}(\pi)$. Заметим, что при $\dot{\bullet}_{d}=\dot{\bullet}\left(\stackrel{\circ}{X}_{d}=\stackrel{\circ}{G}\right)$ имеем $\stackrel{\circ}{f}=\stackrel{\bullet}{c}(f)\left(\dot{f}_{d}=\stackrel{\circ}{c}(d)\right)$.

Если $\left\{C_{k}\right\}_{k \in G_{v}}-$ морфизм из представления $\pi$ в представление $\tilde{\pi}$, то $\stackrel{\circ}{F}_{d f}\left(\left\{C_{k}\right\}\right)=\stackrel{\circ}{F}_{X_{d}, \delta}\left(\left\{C_{k}\right\}\right), \dot{F}_{d f}\left(\left\{C_{k}\right\}\right)=\dot{\circ}_{X_{d}, \delta}\left(\left\{C_{k}\right\}\right)$.

Из формул $(5),(6)$ и $(7)$ следует, что функтор $F_{d f}$ строгий и полный (в смысле [20]). Кроме того, легко видеть, что $\stackrel{\circ}{F}_{c(d), \stackrel{\circ}{f}_{d}} \stackrel{\circ}{F}_{d f} \cong \operatorname{Id~и~} \stackrel{\circ}{F}_{d f} \stackrel{\circ}{F}_{c(d), \stackrel{\circ}{f}_{d}} \cong \operatorname{Id~и~}$ функтор $\stackrel{\circ}{F}_{d f}$ осуществляет эквивалентность категорий. Справедливо и аналогичное утверждение о $\dot{F}_{d f}$.

Определим категорию $\operatorname{Rep}(G, \amalg)$ следующим образом: $\operatorname{Ob} \operatorname{Rep}(G, \amalg)=$ $\coprod_{(d, f) \in S} \operatorname{Ob} \operatorname{Rep}(G, d, f)$, а морфизмы между объектами из $\operatorname{Ob} \operatorname{Rep}\left(G, d_{1}, f_{1}\right)$ и $\operatorname{Ob} \operatorname{Rep}\left(G, d_{2}, f_{2}\right)$ совпадают с морфизмами в $\operatorname{Rep}\left(G, d_{1}, f_{1}\right)$ при $\left(d_{1}, f_{1}\right)=\left(d_{2}, f_{2}\right)$ и отсутствуют при $\left(d_{1}, f_{1}\right) \neq\left(d_{2}, f_{2}\right)$.

Определим такие полные подкатегории в $\operatorname{Rep}(G, \amalg)$ : множество объектов категории $\operatorname{Rep}_{\circ}(G, \amalg)$ есть $\coprod_{(d, f) \in S_{\circ}} \operatorname{Ob} \operatorname{Rep}(G, d, f)$, а множество объектов категории $\operatorname{Rep}(G, \amalg)$ есть $\coprod_{(d, f) \in S} . \operatorname{Ob}_{\operatorname{Rep}}(G, d, f)$. Функторы $\stackrel{\circ}{F}_{d f}\left(\dot{F}_{d f}\right)$ естественным образом порождают функтор $\stackrel{\circ}{F}(\dot{F})$ на категории $\operatorname{Rep}_{\circ}(G, \amalg)\left(\operatorname{Rep}_{\bullet}(G, \amalg)\right)$, осуществляющий эквивалентность категории с собой.

Таким образом, мы доказали следующее утверждение:

Теорема 2.1. Определены функторы четных и нечетных отражений

$$
\stackrel{\circ}{F}: \operatorname{Rep}_{\circ}(G, \amalg) \rightarrow \operatorname{Rep}_{\circ}(G, \amalg), \quad \stackrel{\circ}{F}: \operatorname{Rep}_{\bullet}(G, \amalg) \rightarrow \operatorname{Rep}_{\bullet}(G, \amalg),
$$


осуществляющие эквивалентности категории с собой и следующие эквивалентности полных подкатегорий:

$$
\begin{aligned}
& \stackrel{\circ}{F}: \operatorname{Rep}_{\circ}(G, d, f) \rightarrow \operatorname{Rep}_{\circ}\left(G, \stackrel{\circ}{c}(d), \stackrel{\circ}{f}_{d}\right) \quad n p u(d, f) \in S_{\circ}, \\
& \dot{F}: \operatorname{Rep}_{\bullet}(G, d, f) \rightarrow \operatorname{Rep}_{\bullet}\left(G, \dot{c}(d), \dot{f}_{d}\right) \quad n p u(d, f) \in S_{\bullet} .
\end{aligned}
$$

При этом $(\stackrel{\circ}{F})^{2} \cong \mathrm{Id},(\stackrel{\bullet}{F})^{2} \cong \mathrm{Id}$.

Пусть $g \in G_{v}$ и $\Pi_{g}$ - простейшее представление графа $G: \Pi_{g}(g)=\mathbb{C}$, $\Pi_{g}(a)=0$ при $a \neq g, a \in G_{v}$. Характеры представлений $\Pi_{g}$ будем обозначать через $f_{g}$. Имеем $f_{g}(g)=0$, и будем предполагать при этом, что $f_{g}(a)>0$ при $a \neq g$.

Для простейшего представления $\Pi_{g}$ имеем $d_{g}(g)=1, d_{g}(a)=0$ при $a \neq g$.

Объекты $\left(\Pi_{g}, f_{g}\right)$ будем называть простейшими объектами категории $\operatorname{Rep}(G, \amalg)$. Если $g \in \stackrel{\bullet}{G}(g \in \stackrel{\circ}{G})$, то $\left(\Pi_{g}, f_{g}\right) \in \operatorname{ObRep}_{\circ}(G, \amalg)\left(\left(\Pi_{g}, f_{g}\right) \in\right.$ Ob Rep. $(G, \amalg))$.

\section{§3. Преобразования Кокстера}

Обозначим через $B$ квадратичную форму на пространстве $V_{G}$, определяемую формулой $B(x)=\sum_{a \in G_{v}} x_{a}^{2}-\sum_{\gamma_{a b} \in G_{e}} x_{a} x_{b}$, а через $\langle$,$\rangle соответствую-$ щую симметричную билинейную форму. Форма $B(x)$ называется формой Tuтса графа $G$.

В предложениях 3.1-3.3 сформулированы известные результаты (см., например, [1]), которые понадобятся нам в дальнейшем.

Для каждого $a \in G_{v}$ обозначим через $\bar{a}$ вектор в $V_{G}$, такой, что $(\bar{a})_{b}=0$ при $a \neq b$ и $(\bar{a})_{a}=1$.

ПРЕДЛОЖЕНИЕ 3.1. 1. Пусть $a \in G_{v} ;$ тогда $\sigma_{a}(x)=x-2\langle\bar{a}, x\rangle \bar{a}, \sigma_{a}^{2}=1$.

2. Группа $W$, порождаемая отражениями $\sigma_{a}$, сохраняет иелочисленную решетку в $V_{G}$ и сохраняет квадратичную форму $B$.

3. Если борма $B$ положстельно определена, то группа $W$ конечна.

4. Форма $B$ положстельно определена для графов $A_{n}, D_{n}, E_{6}, E_{7}, E_{8}$ (графов Дынкина) и только для них.

Вектор $x \in V_{G}$ называется (вещественным) корнем, если для некоторых $a \in G_{v}$ и $w \in W$ имеем $x=w \bar{a}$. Векторы $\bar{a}$ называются простыми корнями. Корень $x$ называется положительным, если $x>0$.

ПРЕДЛОЖЕНИЕ 3.2. 1. Если $x$ - коренъ, то $x$ - целочисленный вектор $u$ $B(x)=1$.

2. Если $x-$ корень, то $и-x-$ корень.

3. Если $x$ - корень, то либо $x>0$, либо $(-x)>0$.

ПрЕДЛОЖЕНИЕ 3.3. Если форма $B$ для графа $G$ положительно определена, то

1) преобразование с в пространстве $V_{G}$ не имеет ненулевых инвариантнъх векторов;

2) если $x \in V_{G}, x \neq 0$, то для некоторого натурального $k$ вектор $c^{k} x$ не положителен.

Вернемся к локально скалярным представлениям графа $G$ в категории гильбертовых пространств. 
Лемма 3.4. Если $G$ - граф Дынкина $u d=\left(d_{a}\right)$ - его положительный не простой корень, то d получается из простого корня последовательностью четных и нечетных преобразований Кокстера.

ДокАЗАТЕЛЬСтво. Заметим сначала, что если $x$ - положительный корень, а $\stackrel{\circ}{c}(x)(\stackrel{\bullet}{c}(x))-$ неположительный, то $x-$ простой корень. Действительно, пусть $-\stackrel{\circ}{c}(x)>0$ (предложение $3.2(3))$. При $a \in \dot{G}$ имеем $(\stackrel{\circ}{c}(x))_{a}=x_{a}=0$. Если $x_{b}>0, x_{g}>0(b, g \in \stackrel{\circ}{G})$, то $\left(\sigma_{g}(x)\right)_{g}<0$, а $\left(\sigma_{g}(x)\right)_{b}>0$, что противоречит предложению $3.2(3)$.

Пусть $m-$ наименьшее натуральное число со свойством $c^{m}(d) \notin V_{G}^{+}$(такое $m$ найдется по предложению 3.3). Применяя к $d$ последовательно нечетные и четные преобразования Кокстера, мы получим положительный корень $\widetilde{d}$, такой, что следующий вектор будет отрицательным. Тогда $\widetilde{d}$ будет простым корнем. Пусть $\widetilde{d}=\stackrel{\bullet}{c}_{k}(d)$ (или $\left.d=\stackrel{\circ}{c}_{k}(d)\right)$; тогда $d=\stackrel{\bullet}{c}_{k}^{-1}(\widetilde{d})$ (или $d=\stackrel{\circ}{c}_{k}^{-1}(\widetilde{d})$ ), и лемма доказана.

Лемма 3.5. Пусть $\pi \in \operatorname{Rep}(G), a \in G_{v}, d(a) \neq 0, f(a)=0$. Тогда $\pi=\pi_{a} \oplus \pi^{\prime}$, əде $G^{\pi_{a}}=a, a G^{\pi^{\prime}} \not \supset$ а (не исключено, что $\pi^{\prime}=0$ ).

Действительно, так как $f(a)=0$, то $\Gamma_{a b}=0$ при $\gamma_{a b} \in \bar{M}(a)$, а так как $d(a) \neq 0$, то $\pi=\pi_{a} \oplus \pi^{\prime}$.

ПредлОЖенИЕ 3.6. Все локально скалярные представления графа Дынкина $G$ в категории гилъбертовых пространств дискретны.

ДокАЗАТЕЛЬСТво проведем индукцией по $n=\left|G_{v}\right|$. При $n=1$ утверждение тривиализируется (сепарабельное гильбертово пространство есть ортогональная прямая сумма, конечная либо бесконечная, одномерных пространств). Пусть утверждение доказано для графов Дынкина с числом вершин $\leqslant n-1$. Пусть $n>1$ и $\pi \in \mathrm{Ob} \operatorname{Rep}(G)$. Если $G^{\pi} \neq G_{v}$, то можно от $\pi$ перейти к представлению $\widetilde{\pi}$ графа $\widetilde{G}$ с меньшим числом вершин $\left(\widetilde{G}_{v}=G^{\pi}\right)$ и воспользоваться предположением индукции. Пусть $G^{\pi}=G_{v}$. Предположим, что $f \in\left\{f_{\pi}\right\}$, $f(g)=0, g \in G_{v}$. Тогда по лемме $3.5 \pi=\pi_{g} \oplus \pi^{\prime}$, и мы снова можем воспользоваться предположением индукции. Пусть $G^{\pi}=G_{v}$ и $f(a)>0$ при $a \in G_{v}$. Применяя к $(\pi, f)$ поочередно функторы $\stackrel{\circ}{F}_{G}, \stackrel{\bullet}{F}_{G}$, с учетом (9) получим, что

a) либо $c^{k}(f)$ положителен при всех $k$,

b) либо после применения функтора нарушится одно из условий $G^{\tilde{\pi}}=G_{v}$, $\widetilde{f}(a)>0$ при $a \in G_{v}$.

Случай а) противоречит предложению 3.3 ; в случае b) дело сводится к уже рассмотренной ситуации.

ПрЕДЛОЖЕНИЕ 3.7. Любое локалъно скалярное неразложимое представление $\pi$ графа Дынкина $G$ получается из некоторого простейшего представления $\Pi_{g}$ с помощью функторов четных и нечетных отражений. Более точно: если $(\pi, f) \in \widetilde{\operatorname{Rep}}(G, \amalg)$, то существует последовательность $\left(\pi^{(k)}, f^{(k)}\right)$ обгектов категории $\widetilde{\operatorname{Rep}}(G, \amalg), k=0, \ldots, n$, такая, что $\left(\pi^{(0)}, f^{(0)}\right)=\left(\Pi_{g}, f_{g}\right)$ (пусть, для определенности, $g \in \stackrel{\circ}{G})$ u $\dot{F}\left(\pi^{(k-1)}, f^{(k-1)}\right)=\left(\pi^{(k)}, f^{(k)}\right)$ nри нечетном $k$, а при $k$ четном $\stackrel{\circ}{F}\left(\pi^{(k-1)}, f^{(k-1)}\right)=\left(\pi^{(k)}, f^{(k)}\right), k=1, \ldots, n, u\left(\pi^{(n)}, f^{(n)}\right)$ $=(\pi, f)$. 
ДокаЗАТЕЛЬСтво. Пусть $\pi$ имеет размерность $d$ и характер $f$. Пусть $n-$ минимальное число, такое, что $\left\{\stackrel{\circ}{c}_{n}(d), \dot{c}_{n}(d)\right\} \not \subset V_{G}^{+}$. Будем $n$ называть высотой локально скалярного представления $\pi$.

Предложение докажем индукцией по $n$. Пусть $n=1$; тогда $f(g)=0$ для некоторого $g \in G^{\pi} \cap \stackrel{\circ}{G}$ (в противном случае применим к $\pi$ функтор $\stackrel{\circ}{F}_{X, \delta}$, где $X=G^{\pi}$, и получим $\left.d\left(\stackrel{\circ}{F}_{X, \delta}(\pi)\right)=\stackrel{\circ}{c}(d(\pi)) \in V_{G}^{+}\right)$. Тогда из неразложимости $\pi$ и леммы 3.5 следует, что $G^{\pi}=\{g\}$, т. е. $\pi=\Pi_{g},(\pi, f)$ - простейший объект.

Пусть утверждение доказано для локально скалярных представлений высоты $\leqslant n-1$ и $\pi$ имеет высоту $n \geqslant 1$. По лемме 3.5 имеем $(\pi, f) \in \operatorname{Rep}_{\circ}(G, \amalg)$ и $(\pi, f) \in \operatorname{Rep}_{\bullet}(G, \amalg)$, и к паре $(\pi, f)$ применимы оба функтора $\stackrel{\circ}{F}$ и $\stackrel{\bullet}{F}$. Будем применять тот функтор, который даст новую пару с представлением меньшей высоты. Дальше воспользуемся предположением индукции.

Из теоремы 2.1 и предложения 3.7 вытекает

СлЕДСТвиЕ 3.8. В категории $\widetilde{\operatorname{Rep}}(G, d, f)$ все объекты эквивалентны.

Пусть $\bar{a}-$ простой корень из $V_{G}$ и для определенности $a \in \stackrel{\circ}{G}$. Тогда $c^{l(a)}(\bar{a})>0$ для некоторого натурального числа $l(a)$, а $c^{l(a)+1}(\bar{a}) \ngtr 0$. Будем считать, что $l(a)$ - наименьшее число с таким свойством. Как мы уже указывали ранее, переход от положительного корня $x$ к неположительному корню $y$ при помощи чередующихся преобразований $\stackrel{\bullet}{c}$ и $\stackrel{\circ}{c}$ совершается прохождением через простой корень. Наименьшее не равное нулю число шагов, за которое простой корень $\bar{a}$ переводится в простой корень $\bar{b}$ преобразованиями $\stackrel{\bullet}{c} \stackrel{\circ}{c}$, обозначим через $h(a)$ : $\dot{c}_{h(a)}(\bar{a})=\bar{b}$. Имеются две возможности:

a) $h(a)=2 l(a)$. В этом случае вершина $b$ нечетна (т.е. $b \in \dot{G}$ ). В цепочке корней $\bar{a}, \dot{c}(\bar{a}), \dot{c}_{2}(\bar{a}), \ldots, \dot{c}_{2 l(a)}(\bar{a})=\bar{b}$ любой из корней получается из простого корня $(\bar{a}$ либо $\bar{b})$ преобразованиями $\stackrel{\bullet}{c}, \stackrel{\circ}{c}$ за число шагов $\leqslant h(a) / 2$. Средний в цепочке корень будем получать, для определенности, из нечетного простого корня (за $h(a) / 2=l(a)$ шагов).

b) $h(a)=2 l(a)+1, \dot{c}_{2 l(a)+1}(\bar{a})=\bar{b}$. В этом случае $\bar{b}$ имеет ту же четность, что и $\bar{a}$ (в частности, возможно, что $\bar{a}=\bar{b}$ ). Любой корень в цепочке $\bar{a}, \dot{c}(\bar{a}), \ldots, \dot{c}_{2 l(a)+1}(\bar{a})=\bar{b}$ получается из простого корня $(\bar{a}$ либо $\bar{b})$ преобразованиями $\stackrel{\bullet}{c}, \stackrel{\circ}{c}$ за число шагов $<h(a) / 2$.

Пусть $S_{G}$ - множество простых корней графа $G, u_{g}(a) \in\left(\mathbb{R}^{+}\right)^{G_{v} \backslash\{g\}}$ (по функции $u_{g}$ мы будем строить характер $f_{g}$, полагая $f_{g}(a)=u_{g}(a)$ при $a \in$ $G_{v} \backslash\{g\}$ и $\left.f_{g}(g)=0\right)$. Пусть $N_{G}=\left\{\left(\bar{a}, k, u_{a}\right) \mid \bar{a} \in S_{G} ; k \leqslant h(a) / 2\right.$ для $a \in \dot{G}$ и $k<h(a) / 2$ для $\left.a \in \stackrel{\circ}{G} ; u_{a} \in\left(\mathbb{R}^{+}\right)^{G_{v} \backslash\{a\}}\right\}$, a Ind $G$ - множество неразложимых локально скалярных представлений графа $G$, определенных с точностью до унитарной эквивалентности.

По простому корню $\bar{g}$ и функции $u_{g}(a)$ цепочкой отражений Кокстера за $k$ шагов мы получим из простейшего объекта $\left(\Pi_{g}, f_{g}\right)$ объект $(\pi, f)$ категории $\widetilde{\operatorname{Rep}}(G, \amalg)(\pi-$ неразложимое в $\operatorname{Rep}(G)$ представление из совокупности Ind $G)$. 
Таким образом, мы определили отображение

$$
\varphi: N_{G} \rightarrow \operatorname{Ind} G .
$$

Все неразложимые локально скалярные представления графа Дынкина получаются таким образом (см. предложение 3.7$)$.

Пусть $\overline{\operatorname{Ind}} G$ - подмножество точных представлений в Ind $G, T_{G}$ - подмножество тех простых корней $\bar{a}$ и $L_{a}-$ множество тех значений $k$, для которых $\left(\bar{a}, k, u_{a}\right) \in \varphi^{-1}(\overline{\operatorname{Ind}} G)$.

Имеет место

Tеорема 3.9. Отображение $\varphi: N_{G} \rightarrow$ Ind $G$ есть сюоргекия, причем каждое точное представление $\pi$ из Ind $G$ имеет единственный $\varphi$-прообраз; для неточного $\pi$ вектор $\bar{a}$ и число $k$ в $\varphi^{-1}(\pi)$ определяются однозначно, а функиия $u_{a}$ не однозначно.

ДокАЗАТЕЛЬСтво получим прямым подсчетом для графов Дынкина $E_{6}, E_{7}$, $E_{8}$ и по индукции для графов $A_{n}$ и $D_{n}$.

1. Для графа $A_{n}$

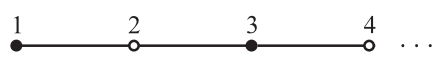

$h(i)=n$ для $i=1, \ldots, n$;

при $n=2 m+1$ либо $n=2 m$ с четным $m$ имеем $T_{A_{n}}=\{\overline{m+1}\}, L_{m+1}=\{m\}$; при $n=2 m$ с нечетным $m$ имеем $T_{A_{n}}=\{\bar{m}\}, L_{m}=\{m\}$.

2. Для графа $D_{n}(n \geqslant 4)$

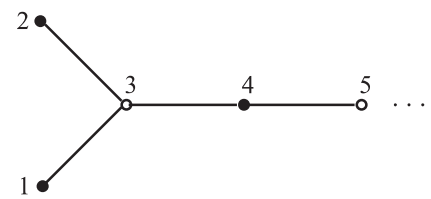

$h(i)=2 n-3, i=1, \ldots, n$;

при $n=2 m+1$ имеем $T_{D_{n}}=\{\overline{3}, \overline{4}, \ldots, \overline{m+2}\}, L_{3}=\{2 m-2,2 m-1\}, L_{4}=$ $\{2 m-3,2 m-2\}, \ldots, L_{m+1}=\{m, m+1\}, L_{m+2}=\{m\}$;

при $n=2 m$ имеем $T_{D_{n}}=\{\overline{3}, \overline{4}, \ldots, \overline{m+1}\}, L_{3}=\{2 m-3,2 m-2\}, \ldots, L_{m+1}=$ $\{m-1, m\}$.

3. Для графа $E_{6}$

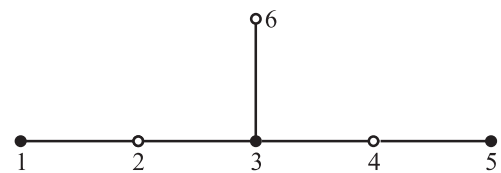

$h(i)=11, i=1, \ldots, 6$;

$T_{E_{6}}=\{\overline{2}, \overline{3}, \overline{4}, \overline{6}\}, L_{2}=\{5\}, L_{3}=\{2,3,4,5\}, L_{4}=\{5\}, L_{6}=\{4\}$.

4. Для графа $E_{7}$

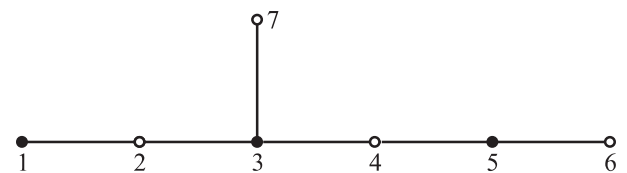

$h(i)=17, i=1, \ldots, 7$;

$T_{E_{7}}=\{\overline{2}, \overline{3}, \overline{4}, \overline{5}, \overline{7}\}, L_{2}=\{5,6,7\}, L_{3}=\{3,4,5,6,7,8\}, L_{4}=\{3,6,7,8\}, L_{5}=$ $\{7\}, L_{7}=\{4,8\}$. 
5. Для графа $E_{8}$

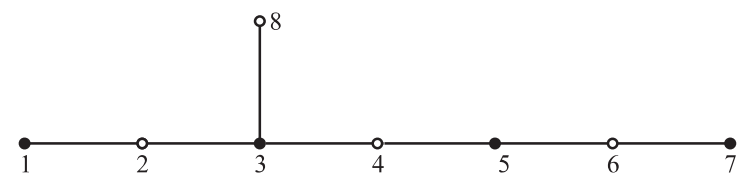

$h(i)=29, i=1, \ldots, 8$;

$T_{E_{8}}=\{\overline{1}, \overline{2}, \overline{3}, \overline{4}, \overline{5}, \overline{6}, \overline{8}\}, L_{1}=\{7,12,13\}, L_{2}=\{5-8,11-14\}, L_{3}=\{4-14\}$, $L_{4}=\{3,4,7-14\}, L_{5}=\{8-13\}, L_{6}=\{11,12\}, L_{8}=\{9,10,13,14\}$.

Таким образом, в процессе доказательства теоремы 3.9 мы доказали

СлеДСтвиЕ 3.10. Для любого графа Дынкина $G$ существует взаимно однозначное соответствие между точными неразложимыми локально скалярными представлениями графа $G$ и тройками $\left(\bar{a}, k, u_{a}\right) \in N_{G}$, где $\bar{a} \in T_{G}, k \in L_{a}$.

Рассмотрим в пространстве $V_{G}$ скалярное произведение $x y$, считая, что векторы $\bar{a}\left(a \in G_{v}\right)$ образуют в $V_{G}$ ортонормированный базис. Таким образом, если $x=\left(x_{a}\right), y=\left(y_{a}\right)$, то $x y=\sum_{a \in G_{v}} x_{a} y_{a}, x=\sum_{a \in G_{v}} x_{a} \bar{a}=\sum_{a \in \dot{G}} x_{a} \bar{a}+\sum_{b \in G} x_{b} \bar{b}$.

Введем обозначения $x^{\bullet}=\sum_{a \in \dot{G}} x_{a} \bar{a}, x^{\circ}=\sum_{b \in G} x_{b} \bar{b}\left(x=x^{\bullet}+x^{\circ}\right)$.

Учитывая п. 1) предложения 3.1, получим, что $\dot{c}(x)=x-2 \sum_{a \in \dot{G}}\langle\bar{a}, x\rangle \bar{a}=$ $x-2 \sum_{a \in \dot{G}}\left\langle\bar{a}, x^{\bullet}+x^{\circ}\right\rangle \bar{a}=x-2 \sum_{a \in \dot{G}}\left\langle\bar{a}, x^{\bullet}\right\rangle \bar{a}-2 \sum_{a \in \dot{G}}\left\langle\bar{a}, x^{\circ}\right\rangle \bar{a}=x-2 x^{\bullet}-$ $2 \sum_{a \in \dot{G}}\left\langle\bar{a}, x^{\circ}\right\rangle \bar{a}=x^{\circ}-x^{\bullet}-2 \sum_{a \in \dot{G}}\left\langle\bar{a}, x^{\circ}\right\rangle \bar{a}$. (Здесь мы использовали тот факт, что $\langle\bar{a}, \bar{a}\rangle=1$ и $\left\langle\bar{a}, \bar{a}^{\prime}\right\rangle=0$ при $a \neq a^{\prime}, a, a^{\prime} \in \dot{G}$.)

Окончательно,

$$
\dot{c}(x)=x^{\circ}-x^{\bullet}-2 \sum_{a \in \dot{G}}\left\langle\bar{a}, x^{\circ}\right\rangle \bar{a} .
$$

Аналогично,

$$
\stackrel{\circ}{c}(x)=x^{\bullet}-x^{\circ}-2 \sum_{b \in \stackrel{\circ}{G}}\left\langle\bar{b}, x^{\bullet}\right\rangle \bar{b}
$$

Докажем

ПреДЛОЖЕНИЕ 3.11. 1. Если $x, y \in V_{G} u x^{\bullet} y^{\bullet}=x^{\circ} y^{\circ}$, mo $[\stackrel{\bullet}{c}(x)]^{\bullet} \cdot[c(y)]^{\bullet}=$ $[\stackrel{\bullet}{c}(x)]^{\circ} \cdot[\stackrel{\circ}{c}(y)]^{\circ}$

2. Если $\pi$ - конечномерное $f$-представление графа $G$ размерности $d(a)$, $a \in G_{v}, \operatorname{mo}^{\bullet} \cdot d^{\bullet}=f^{\circ} \cdot d^{\circ}$

ДокАЗАТЕЛЬСтво. 1. Из равенств (10) следует, что $[\stackrel{\bullet}{c}(x)]^{\bullet} \cdot\left[{ }^{\circ}(y)\right]^{\bullet}=\left(-x^{\bullet}-2 \sum_{a \in \dot{G}}\left\langle\bar{a}, x^{\circ}\right\rangle \bar{a}\right) y^{\bullet}=-x^{\bullet} y^{\bullet}-2 \sum_{a \in \dot{G}}\left\langle\bar{a}, x^{\circ}\right\rangle y_{a}^{\bullet}=-x^{\bullet} y^{\bullet}-2\left\langle y^{\bullet}, x^{\circ}\right\rangle$, $[\bullet(x)]^{\circ} \cdot[\stackrel{\circ}{c}(y)]^{\circ}=x^{\circ} \cdot\left(-y^{\circ}-2 \sum_{b \in G}\left\langle\bar{b}, y^{\bullet}\right\rangle \bar{b}\right)=-x^{\circ} y^{\circ}-2 \sum_{b \in G}\left\langle\bar{b}, y^{\bullet}\right\rangle x_{b}^{\circ}=-x^{\circ} y^{\circ}-2\left\langle x^{\circ}, y^{\bullet}\right\rangle$, и так как $x^{\bullet} y^{\bullet}=x^{\circ} y^{\circ}$, a $\left\langle y^{\bullet}, x^{\circ}\right\rangle=\left\langle x^{\circ}, y^{\bullet}\right\rangle$, то $\left[\stackrel{\bullet}{c}(x)^{\bullet} \cdot\left[{ }^{\circ}(y)\right]^{\bullet}=[\stackrel{\bullet}{c}(x)]^{\circ} \cdot[\stackrel{\circ}{c}(y)]^{\circ}\right.$.

2. Из условия локальной скалярности $\sum_{b \in M_{a}} \Gamma_{a b} \Gamma_{b a}=\alpha_{a} I_{H_{a}}$ для конечномерного представления следует, что $\sum_{b \in M_{a}} \operatorname{Tr}\left(\Gamma_{a b} \Gamma_{b a}\right)=\alpha_{a} d_{a}\left(\alpha_{a}=f(a)\right.$, $\left.d_{a}=d(a), a \in G_{v}\right)$. 
Просуммировав такие равенства сначала по нечетным вершинам, а потом по четным, мы получим

$$
f^{\bullet} d^{\bullet}=\sum_{\left\{\gamma_{a b}, \gamma_{b a}\right\} \subset G_{e}} \operatorname{Tr}\left(\Gamma_{a b} \Gamma_{b a}\right)=\sum_{a \in G_{v}} \alpha_{a} d_{a}=f^{\circ} d^{\circ} .
$$

\section{§4. Аналог теоремы Габриеля}

ПрЕДЛОЖЕНИЕ 4.1. Если граф $G$ есть один из расширенных графов Дынкина $\widetilde{A}_{n}, \widetilde{D}_{n}, \widetilde{E}_{6}, \widetilde{E}_{7}, \widetilde{E}_{8}$, то у него размерности неразложимых локалъно скалярных представлений не ограничены в совокупности, так что $G$ не конечно представим в $\mathscr{H}$.

ДокАзАТЕЛЬСтво. Из теории корней расширенных графов Дынкина следует, что если $g \in \stackrel{\circ}{G}$, то $c^{t}(\bar{g}) \in V_{G}^{+}$при $t>1$ и $c^{t}(\bar{g})$ не есть простой корень (а если $g \in \dot{G}$, то $c^{-t}(\bar{g}) \in V_{G}^{+}$при $t>1$ и $c^{-t}(\bar{g})$ не есть простой корень) (см. также [21]), откуда вытекает, что $c^{t_{1}}(\bar{g}) \neq c^{t_{2}}(\bar{g})$ при $t_{1} \neq t_{2}$.

Тогда, зафиксировав $g \in \stackrel{\circ}{G}$ и простейший объект $\left(\Pi_{g}, f\right) \in \operatorname{Rep}(G, \amalg)$, получим бесконечную последовательность объектов ... $\stackrel{\circ}{F} \dot{F}\left(\Pi_{g}, f\right)$, у которых размерности представлений различны.

Укажем на связь между неразложимыми представлениями колчанов, граф которых есть схема Дынкина, и локально скалярными представлениями этих графов.

Пусть $\pi^{\Lambda}-$ конечномерное представление колчана $Q$ над полем $\mathbb{C}$. Если пространства представления гильбертовы (т. е. унитарны, так как они конечномерны), то представление $\pi^{\Lambda}$ может быть естественным образом продолжено до представления $\pi$ графа $G=G(Q)$ в категории гильбертовых пространств.

Если $\pi^{\Lambda}$ эквивалентно в $\operatorname{Rep}(Q, \mathbb{C})$ представлению $\widetilde{\pi}^{\Lambda}$, такому, что продолженное представление $\widetilde{\pi}$ графа $G$ локально скалярно, будем говорить, что представление $\pi^{\Lambda}$ колчана $Q$ унитаризуемо.

ПредЛОЖенИЕ 4.2. Граф $G$ есть граф Дынкина тогда и только тогда, когда при $G=G(Q)$ любое конечномерное неразложимое представление $\pi^{\Lambda}$ колчана $Q$ унитаризуемо.

ДокАзАтельство. Если граф $G$ есть граф Дынкина, то размерность $d(a)$ представления $\pi^{\Lambda}$ есть корень, который может быть получен из некоторого простого корня $\bar{g}$ преобразованиями $\stackrel{\bullet}{c}$ и $\stackrel{\circ}{c}$. Из простейшего представления $\Pi_{g}$ в этой же размерности $d(a)$ функторами $\dot{F}$ и $\stackrel{\circ}{F}$ может быть получено локально скалярное представление $\tilde{\pi}$. Представления $\pi^{\Lambda}$ и $\widetilde{\pi}^{\Lambda}$ колчана $Q$ эквивалентны в $\operatorname{Rep}(Q, \mathbb{C})$, так как для графов Дынкина в размерности $d(a)$ существует единственное с точностью до эквивалентности неразложимое представление $[1,2]$.

Условие унитаризуемости можно было бы переформулировать и так: для представления $\pi^{\Lambda}$ колчана $Q$ можно так ввести скалярное произведение в пространствах представления, что продолженное представление графа $G$ в категории гильбертовых пространств будет локально скалярным.

Мы докажем вторую часть утверждения предложения 4.2 , если покажем, что для любого расширенного графа Дынкина $G$ (а значит, и для $G^{\prime} \supset G$ ) и любого 
колчана $Q$, такого, что $G(Q)=G$, найдется неразложимое представление $\pi^{\Lambda}$ колчана $Q$, которое будет не унитаризуемым.

Если $(d(a))_{a \in G_{v}}$ - минимальный мнимый корень графа $G$ (т. е. минимальный положительный корень уравнения $B_{G}(x)=0$ ), то такое представление найдется в размерности $2 d(a)$.

Пусть для определенности ориентация колчана $Q$ будет такой, что для каждой четной вершины $a$ все инцидентные ей стрелки «входят» в вершину, а для нечетной - «выходят» из нее.

Если все инцидентные вершине $а$ стрелки «входят» в эту вершину, то найдется (см., например, [10]) представление $\pi^{\Lambda}$, для которого

$$
\pi^{\Lambda}\left(\gamma_{a b}\right)=\left[\begin{array}{c|c}
\pi_{1}^{\Lambda}\left(\gamma_{a b}\right) & X\left(\gamma_{a b}\right) \\
\hline 0 & \pi_{2}^{\Lambda}\left(\gamma_{a b}\right)
\end{array}\right]
$$

$\pi^{\Lambda}(a)=H_{1}^{(a)} \oplus H_{2}^{(a)}, \pi^{\Lambda}(b)=H_{1}^{(b)} \oplus H_{2}^{(b)}, \pi_{1}^{\Lambda}\left(\gamma_{a b}\right): H_{1}^{(b)} \rightarrow H_{1}^{(a)}, \pi_{2}^{\Lambda}\left(\gamma_{a b}\right): H_{2}^{(b)} \rightarrow$ $H_{2}^{(a)}, d_{a}=\operatorname{dim} H_{1}^{(a)}=\operatorname{dim} H_{2}^{(a)}$ для $a \in G_{v}$.

Предположим, что представление $\pi^{\Lambda}$ унитаризуемо, т. е. в пространствах представления можно так ввести скалярное произведение, что продолженное представление $\pi$ графа $G$ станет локально скалярным.

Выбирая ортогональный базис в пространствах $H_{1}^{(a)}$ и дополняя его до ортогонального базиса в $H_{1}^{(a)} \oplus H_{2}^{(a)}$, мы получим новые матрицы представления $\tilde{\pi}^{\Lambda}$, остающиеся приведенными и удовлетворяющие условию локальной скалярности. Не умаляя общности, будем считать, что уже само представление $\pi^{\Lambda}$ обладает этим свойством.

Пусть $y_{k l}^{(a b)}$ - матричные элементы матрицы $\pi_{1}^{\Lambda}\left(\gamma_{a b}\right)$. Тогда сумма $\sum_{k, l} \bar{y}_{k l}^{(a b)} y_{k l}^{(a b)}$ есть сумма квадратов длин столбцов матрицы $\pi_{1}^{\Lambda}\left(\gamma_{a b}\right)$, а значит,

$$
A \equiv \sum_{\gamma_{a b}} \sum_{k, l} \bar{y}_{k l}^{(a b)} y_{k l}^{(a b)}=\sum_{b \in \dot{G}} d_{b} \alpha_{b} .
$$

Если $x_{k l}^{(a b)}$ и $z_{k l}^{(a b)}$ - матричные элементы матриц $X\left(\gamma_{a b}\right)$ и соответственно $\pi_{2}^{\Lambda}\left(\gamma_{a b}\right)$, то для оставшихся столбцов матриц представления имеем

$$
C+B \equiv \sum_{\gamma_{a b}} \sum_{k, l} \bar{x}_{k l}^{(a b)} x_{k l}^{(a b)}+\sum_{\gamma_{a b}} \sum_{k, l} \bar{z}_{k l}^{(a b)} z_{k l}^{(a b)}=\sum_{b \in \dot{G}} d_{b} \alpha_{b},
$$

где $C>0$; поэтому $A>B$.

Аналогично, действия со строками (суммирование по четным вершинам) даст неравенство $B>A$. Таким образом, предположение о локальной скалярности представления $\pi$ приводит к противоречию.

Ранее мы определили множества $S, S_{\circ}, S_{\bullet}$. Определим отображение $\stackrel{\circ}{\mathscr{F}}: S_{\circ} \rightarrow$ $S_{\circ}$, для которого $\stackrel{\circ}{\mathscr{F}}(d, f)=(\stackrel{\circ}{c}(d), \stackrel{\circ}{f} d)$, и отображение $\stackrel{\mathscr{F}}{:} S_{\bullet} \rightarrow S_{\bullet}$, для которого $\stackrel{\mathscr{F}}{ }(d, f)=\left(\stackrel{\bullet}{c}(d), \dot{f}_{d}\right)$. Если пара $\underbrace{\ldots \stackrel{\circ}{\mathscr{F}} \mathscr{\mathscr { F }}}_{k \text { раз }}(d, f)$ определена, то будем ее обозначать через $\stackrel{\mathscr{F}}{F}_{k}(d, f)$, а пару $\underbrace{\ldots \stackrel{\bullet}{\mathscr{F}} \mathscr{\mathscr { F }}}_{k \text { раз }}(d, f)$ через $\stackrel{\circ}{\mathscr{F}}_{k}(d, f), k=0,1,2, \ldots ;$ при $k=0$ получаем саму пару $(d, f)$. (Строго говоря, нужно определить подмножества $S_{i}, i \in \mathbb{N}$, следующим образом: $S_{1}=S_{\circ} \cap S_{\bullet}, S \supset S_{1} \supset \cdots \supset S_{k} \supset \cdots$, причем 
$S_{i+1}=\left\{(d, f) \mid \stackrel{\circ}{\mathscr{F}}(d, f) \in S_{i}\right.$ и $\left.\stackrel{\bullet}{\mathscr{F}}(d, f) \in S_{i}\right\}$. Таким образом, $\stackrel{\circ}{\mathscr{F}}$ и $\stackrel{\bullet}{\mathscr{F}}$ определе-

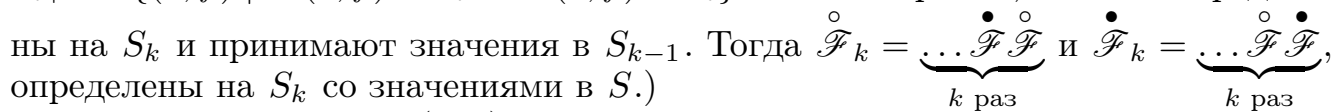

Будем называть пару $(d, f)$ корневой на $G$, если для некоторого $k \in \mathbb{N}_{0}$ имеем $(d, f)=\stackrel{\circ}{\mathscr{F}}_{k}\left(g, f_{g}\right)$ при $g \in \stackrel{\bullet}{G}$ или $(d, f)=\stackrel{\bullet}{\mathscr{F}}_{k}\left(g, f_{g}\right)$ при $g \in \stackrel{\circ}{G}$. Две корневые пары $\left(d, f_{1}\right)$ и $\left(d, f_{2}\right)$ будем называть эквивалентными, если $\left.\left.f_{1}\right|_{X_{d}} \equiv f_{2}\right|_{X_{d}}$. Класс эквивалентности корневой пары $(d, f)$ будем обозначать через $[d, f]$.

ТЕОРема 4.3. Пусть $G$ - связный конечный граф без ииклов.

1. Следующие условия эквивалентны:

(a) графб $G$ есть один из графов Дьнкина $A_{n}, D_{n}, E_{6}, E_{7}, E_{8}$;

(b) граф $G$ конечно представим в $\mathscr{H}$;

(c) всякое конечномерное неразложимое представление колчана $Q$ унитаризуемо.

2. Отображсение $\pi \rightarrow[d(a), f(a)]$, где $d(a)$ - размерность представления $\pi$, a $f(a)$ - его характер, устанавливает взаимно однозначное соответствие межнду классами эквивалентных неразложимых локально скалярных представлений графа $G$ и классами эквивалентных корневых пар графа $G$.

Доказательство. 1. Из (а) следует (b). Действительно, если $G$ - граф Дынкина, то все его представления дискретны по предложению 3.6. Все размерности неразложимых представлений ограничены в совокупности, так как они конечны и их конечное число (размерности неразложимых представлений суть корни графа $G$ по предложению 3.7). Для доказательства конечной представимости графа $G$ в $\mathscr{H}$, очевидно, достаточно показать, что в $\widetilde{\operatorname{Rep}}(G, d, f)$ содержится ровно один с точностью до эквивалентности объект (следствие 3.8) и что два конечномерных представления $\pi$ и $\widetilde{\pi}$ из $\operatorname{Rep}(G)$ унитарно эквивалентны тогда и только тогда, когда они имеют одинаковую размерность $d$, общий характер $f$ и когда пара $(\pi, f)$ эквивалентна $(\tilde{\pi}, f)$ в $\operatorname{Rep}(G, d, f)$.

Из (b) следует (a), так как если $G$ не есть граф Дынкина, то он не конечно представим по предложению 4.1 (он содержит расширенный граф Дынкина).

(а) и (с) эквивалентны по предложению 4.2.

2. Если два локально скалярных представления $\pi_{1}$ и $\pi_{2}$ графа $G$ унитарно эквивалентны и неразложимы (а значит, конечномерны), то очевидно, что их размерности совпадают, а также совпадают (однозначно определенные) на общем носителе их характеры.

Пусть локально скалярные неразложимые представления $\pi_{1}$ и $\pi_{2}$ имеют одинаковую размерность $d$ и одинаковый характер $f$ (на общем носителе характеры совпадают, а вне носителя мы их доопределим одинаковым образом). Тогда $\pi_{1}$ и $\pi_{2}$ имеют одинаковую высоту и $d=\stackrel{\circ}{c}_{t}(\bar{g})$ или $\stackrel{\bullet}{c}_{t}(\bar{g})$. А значит, пары $\left(\pi_{1}, f\right)$, $\left(\pi_{2}, f\right)$ как объекты категории $\widetilde{\operatorname{Rep}}(G, \amalg)$ могут быть одинаково получены из простейшего объекта $\left(\Pi_{g}, f_{g}\right)$ с помощью функторов четных и нечетных отражений (см. доказательство предложения 3.7) и, стало быть, совпадают.

ЗАМЕчАнИЕ 1. Даже для графов Дынкина неразложимые локально скалярные представления не определяются однозначно характером: имеются примеры неразложимых представлений с различными размерностями, но одинаковыми характерами. 
ЗАмечанИе 2. Из наших построений следует, что для графов Дынкина ( по-видимому, только для них) все локально скалярные представления реализуются над полем вещественных чисел.

\section{ЛиТЕРАТУРА}

1. Бернштейн И. Н., Гельфанд И. М., Пономарев В. А. Функторы Кокстера и теорема Габриеля. УМН, 28, вып. 2, 19-33 (1973).

2. Gabriel P. Unzerlegbare Darstellungen I. Manuscripta Math., 6, 71-103 (1972).

3. Назарова Л. А. Представления четвериады. Изв. АН СССР, 31, № 4, 1361-1378 (1967).

4. Gelfand I. M., Ponomarev V. A. Problems of linear algebra and classification of quadruples of subspaces in a finite-dimensional vector space. In: Hilbert space operators and operator algebras (Proc. Internat. Conf., Tihany, Hungary, 1970), Colloq. Math. Soc. Janos Bolyai, Vol. 5, 1972.

5. Кругляк С. А. Представления алгебр, квадрат радикала которых равен нулю. Зап. науч. семинаров ЛОМИ, 28, 60-69 (1972).

6. Назарова Л. А. Представления колчанов бесконечного типа. Изв. АН СССР, сер. матем., 37, 752-791 (1973).

7. Donovan P., Freislich M. R. The representation theory of finite graphs and associated algebras. Carleton Math. Lect. Notes, Vol. 5, 1973, pp. 1-119.

8. Kac V. G. Some remarks on representations of quivers and infinite root systems. Lect. Notes of Math., Vol. 832, 1980, pp. 311-332.

9. Kac V. G. Infinite root systems, representations of graphs and invariant theory, II. J. Algebra, 78, 141-162 (1982).

10. Gabriel P., Roiter A. V. Representations of Finite-Dimensional Algebras. SpringerVerlag, 1997, pp. 1-171.

11. Кругляк С. А., Самойленко Ю. С. Об унитарной эквивалентности наборов самосопряженных операторов. Функц. анализ и его прил., 14, вып. 1, 84-85 (1980).

12. Кругляк С. А. Представления свободных инволютивных колчанов. В кн. Представления и квадратичные формы, Изд. Ин-та математики АН УССР, Киев, 1979, c. $149-151$.

13. Sergeichuk $V$. $V$. Unitary and Euclidean representations of a quiver. Linear Algebra Appl., 278, 37-62 (1998).

14. Ostrovskyi V., Samoilenko Yu. Introduction to the Theory of Representations of Finitely Presented $*$-algebras. I. Representations by bounded operators. Rev. Math. Math. Phys., 11, 1-261 (1999).

15. Ройтер А. В. Боксы с инволюцией. В кн.: Представления и квадратичные формы, Киев, 1979, с. 124-126.

16. Рабанович В. И., Самойленко Ю. С. Когда сумма идемпотентов или проекторов кратна единице. Функц. анализ и его прил., 34, вып. 4, 91-93 (2000).

17. Кругляк С. А., Рабанович В. И., Самойленко Ю. С. О суммах проекторов. Функц. анализ и его прил., 36, вып. 3, 20-35 (2002).

18. Кругляк C. А. Функторы Кокстера для одного класса *-колчанов. Укр. матем. ж., 54, №o6, 789-797 (2002).

19. Kruglyak S. A. Coxeter functors for a certain class of $*$-quivers and $*$-algebras. Methods of Functional Analysis and Topology, 8, No. 4, 49-57 (2002).

20. Басс X. Алгебраическая $K$-теория. Мир, М., 1973.

21. Редчук И. К., Ройтер А. В. Сингулярные локально-скалярные представления колчанов в гильбертовых пространствах и разделяющие функции. Укр. матем. ж., 56, №6, 796-809 (2004). 